\title{
POG Neuroblastoma Stage C
}

National Cancer Institute

\section{Source}

National Cancer Institute. POG Neuroblastoma Stage C. NCI Thesaurus. Code C85426.

The primary tumor is grossly completely or incompletely resected; intracavitary lymph nodes not adherent to the primary tumor are positive microscopically; the liver is not involved microscopically. 